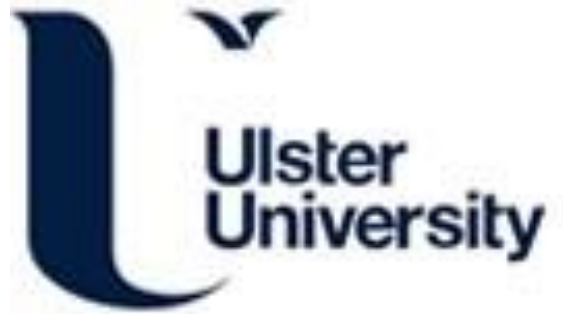

\section{Low-cost process monitoring for polymer extrusion}

Deng, J., Li, K., Harkin-Jones, E., Price, M., Fei, M., Kelly, A., Vera-Sorroche, J., Coates, P., \& Brown, E. (2013). Low-cost process monitoring for polymer extrusion. Transactions of the Institute of Measurement and Control, 382-390. https://doi.org/10.1177/0142331213502696

Link to publication record in Ulster University Research Portal

\section{Published in:}

Transactions of the Institute of Measurement and Control

\section{Publication Status:}

Published (in print/issue): 26/09/2013

DOI:

$10.1177 / 0142331213502696$

\section{Document Version}

Publisher's PDF, also known as Version of record

\section{General rights}

Copyright for the publications made accessible via Ulster University's Research Portal is retained by the author(s) and / or other copyright owners and it is a condition of accessing these publications that users recognise and abide by the legal requirements associated with these rights.

\section{Take down policy}

The Research Portal is Ulster University's institutional repository that provides access to Ulster's research outputs. Every effort has been made to ensure that content in the Research Portal does not infringe any person's rights, or applicable UK laws. If you discover content in the Research Portal that you believe breaches copyright or violates any law, please contact pure-support@ulster.ac.uk. 


\title{
Low-cost process monitoring for polymer extrusion
}

Measurement and Control

2014, Vol. 36(3) 382-390

(c) The Author(s) 2013

Reprints and permissions:

sagepub.co.uk/journalsPermissions.nav

DOI: 10.1 I77/0I4233।213502696

tim.sagepub.com

\author{
Jing Deng', Kang Li ${ }^{2}$, Eileen Harkin-Jones', Mark Price', Minrui Fei ${ }^{3}$, \\ Adrian Kelly ${ }^{4}$, Javier Vera-Sorroche ${ }^{4}$, Phil Coates ${ }^{4}$ and Elaine Brown ${ }^{4}$
}

\begin{abstract}
Polymer extrusion is regarded as an energy-intensive production process, and the real-time monitoring of both energy consumption and melt quality has become necessary to meet new carbon regulations and survive in the highly competitive plastics market. The use of a power meter is a simple and easy way to monitor energy, but the cost can sometimes be high. On the other hand, viscosity is regarded as one of the key indicators of melt quality in the polymer extrusion process. Unfortunately, viscosity cannot be measured directly using current sensory technology. The employment of on-line, in-line or off-line rheometers is sometimes useful, but these instruments either involve signal delay or cause flow restrictions to the extrusion process, which is obviously not suitable for real-time monitoring and control in practice. In this paper, simple and accurate real-time energy monitoring methods are developed. This is achieved by looking inside the controller, and using control variables to calculate the power consumption. For viscosity monitoring, a 'soft-sensor' approach based on an RBF neural network model is developed. The model is obtained through a two-stage selection and differential evolution, enabling compact and accurate solutions for viscosity monitoring. The proposed monitoring methods were tested and validated on a Killion KTS-100 extruder, and the experimental results show high accuracy compared with traditional monitoring approaches.
\end{abstract}

\section{Keywords}

Soft sensor, energy, process modelling, non-linear modelling, polymer extrusion

\section{Introduction}

Due to their low cost, range of properties (e.g. high strengthto-weight ratio, high temperature resistance, high chemical resistance) and ease of processing, polymers are becoming increasingly prevalent as basic materials in many industrial applications. According to a report from the British Plastics Federation (BPF), the UK polymer industry is made up of 7400 companies which produced around 2.5 million tonnes of plastics in 2011, with a turnover of $£ 19$ billion (Davis, 2011). This number will continue to increase as more traditional materials, such as wood, concrete and metal, are substituted with polymers.

The light weight of plastics can help to reduce energy consumption in transportation and related areas. For instance, the Airbus A380 is $22 \%$ carbon fibre reinforced and burns $20 \%$ less fuel per seat than the airline's 747-400 fleet., and for the Boeing 787 Dreamliner, the lighter but strong fuselage gives a $30 \%$ reduction in maintenance cost and a $20 \%$ reduction in fuel consumption compared to similar sized airplanes. (Davis, 2011). The processing of plastics, however, is energyintensive. In the $\mathrm{UK}$, the electricity bill for this purpose amounts to $£ 380$ million per annum. Thus, a reduction in electricity usage of $10 \%$ would result in savings of $£ 38$ million per annum, and a significant reduction in environmental burden.

Generally, the energy consumed during polymer processing can be divided into two aspects: the 'high-level energy management system' and 'low-level machinery control'. For the former, it has been shown that a $30 \%$ reduction in energy use can be achieved by appropriate changes in management, maintenance, and capital investment (Kent, 2008). For the processing machines, energy efficiency relies on the machine geometry and the material being processed (Kelly et al., 2006). However, non-optimal operating settings can also waste energy. These settings include thermal heating, cooling, and the processing speed, for a typical polymer extrusion process.

Single-screw and twin-screw extrusion are the most common types of extrusion in wide usage. In these processes, plastic granules are pushed by a screw moving from the feed zone to the die, and the heat generated by both the shear stress and

\footnotetext{
'School of Mechanical and Aerospace Engineering, Queen's University Belfast, UK

${ }^{2}$ School of Electronics, Electrical Engineering and Computer Science, Queen's University Belfast, UK

${ }^{3}$ School of Mechatronic Engineering and Automation, Shanghai University, China

${ }^{4}$ School of Engineering, Design and Technology, University of Bradford, UK

\section{Corresponding author:}

Jing Deng, School of Electronics, Electrical Engineering and Computer Science, Queen's University Belfast, Ashby Building, Stranmillis Road, Belfast, BT9 5AH, UK.

Email: jdeng0I@qub.ac.uk
} 
barrel heating helps to melt the granules. The extruder motor is obviously an energy-intensive component, and it consumes around one third of the total energy. Its associated power factor is a critical issue for plastic processing companies, as a lower power factor may lead to an undesired penalty (or surcharge). The on-line monitoring of motor power consumption therefore becomes necessary for the investigation of energy efficiency. Such energy monitoring can also provide useful information on the melt stability and the quality of the final product. The use of a power meter (e.g. the HIOKI 3169-21) is of course the easiest way to monitor motor power consumption, including the apparent power, active power, reactive power and the power factor. However, the installation of power meters for each extruder is costly, and mathematical models based on the process settings seem to be an affordable alternative (Abeykoon et al., 2010; Lai and Yu, 2000). However, existing models are highly dependent on the geometry of the extruder and the materials being processed. It is difficult to use the same model on a different machine without re-training. Fortunately, the motor drive provides most of the essential information, which can be used to theoretically calculate the power consumption. For a DC motor, these variables can be the rotational speed and armature current. In this paper, a simple method will be presented for real-time monitoring of the DC motor power consumption in a singlescrew extruder.

Thermal heating is another big consumer of energy in polymer processing. The majority of the thermal energy is used to keep the temperature of the extruder barrel and die at a specific level. Thus, real-time monitoring of the thermal energy also plays an important role in energy efficiency optimization. Similarly, due to the issue of cost and accuracy, power meters and mathematical models are not suitable for this purpose. A more accurate and reliable method based on the controller output will be introduced in this paper.

The main purpose of adjusting the operating settings is to guarantee a consistent melt quality. In industry, the melt pressure near the screw tip is usually accepted as the main indicator of melt quality. This pressure is known to be proportional to the screw speed, it is also slightly affected by the melt temperature and screw geometry, as well as the material being processed. Unstable melt pressure causes fluctuations to the throughput, which results in variations in the quality of the final product. However, previous research has shown that the melt viscosity is probably the best indicator of melt quality (Cogswell, 1981). The main issue with viscosity monitoring is the lack of in-process viscosity measurement sensors. Rheology viscometers are a popular choice when off-line viscosity measurement suits the purpose. For optimizing the operating settings, a real-time monitoring of melt viscosity is required. In this paper, a slit die with a large width-to-height ratio is used to measure the shear stress and a linear model is produced for mass throughput measurement. The viscosity is then obtained from the ratio of shear stress and shear rate. Unfortunately, this method is not applicable to industrial manufacturing lines as it limits the throughput of the melt stream. A low-cost alternative is the previously proposed 'soft-sensor' approach (Liu et al., 2010, 2012; McAfee, 2005). In this paper, the accuracy of the viscosity model is further improved by properly exciting the plan using pseudorandom sequence (PRS) and the integration of differential evolution (DE) into a two-stage selection for radial basis function (RBF) network model construction.

The rest of this paper is therefore organized as follows. Section 2 presents the on-line monitoring of motor power and thermal energy consumption. The viscosity monitoring techniques are introduced in Section 3, where both physical measuring using a slit die and soft-sensing based on a mathematical model are presented. And finally, Section 4 concludes this paper with suggested future work.

\section{On-line energy monitoring}

The real-time monitoring of power consumption at each component is desirable for optimizing the overall energy efficiency. The use of a power meter is the easiest way to achieve this, but the cost is sometimes too high. Mathematical models based on process settings can provide satisfactory accuracy in power monitoring, but it is difficult to apply the same model to different extruders with different geometries and processing materials. In this section, a simple method based on the controllers of thermal heating and motor drive will be presented. All methods were developed and verified on a Killion KTS100 single-screw extruder located at the Polymer Processing Research Centre, Queen's University Belfast.

\section{Monitoring of heating and cooling energy consumption}

The Killion KTS-100 extruder has separate temperature controllers (Eurotherm 808) for each heating zone. The displacement contactors AFM215-303 are used to regulate the heating and cooling. As a result, pulse width modulation (PWM) is incorporated to implement PID (proportional-integral-derivative) control. Moreover, Zones 1-3 (solid conveying, melting, and metering) are fitted with air fan cooling, and their controller output ranges from -100 to 100 instead of $[0,100]$ for heating only control.

Each temperature controller also supports RS-422 serial communication which is then converted to RS-232 to be connected with a computer. Several variables, such as setting point, measured value, and controller outputs, can be directly read or written through this communication. Therefore, by taking the controller outputs and their associated heating and cooling power, their energy consumption can be easily calculated as

$$
\begin{gathered}
P_{\text {thermal }}=\sum_{i=1}^{5} p_{i} u_{i} \\
p_{i}= \begin{cases}p_{\text {heating }} & u_{i} \geq 0 \\
p_{\text {cooling }} & u_{i}<0\end{cases}
\end{gathered}
$$

where $p_{i},(i=1, \ldots 5)$ denotes the $i^{\text {th }}$ heating or cooling power, and $u_{i}$ represents the $i^{\text {th }}$ controller output.

Figure 1 compares the energy consumption measured by the HIOKI 3169-21 power meter and the calculations from controller outputs. It is clear that the calculated value fit the measurements quit well in the first $5 \mathrm{~min}$. After this period, 


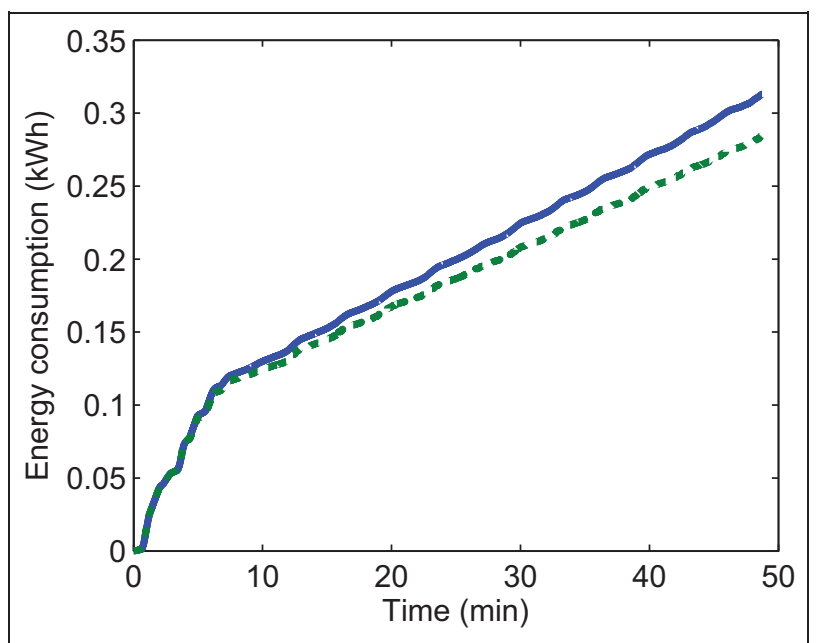

Figure I. Comparison of the thermal energy consumption monitoring by power meter (dotted line) and controller output calculation (solid line).

the calculated value is a little higher than the power meter measurements. This is caused by the lower sampling rate of the power meter. Through serial communication, the maximum sampling rate of this power meter is $1 \mathrm{~Hz}$ while the sampling rate of the temperature controller can be set at $10 \mathrm{~Hz}$. In the first $5 \mathrm{~min}$, the heating works at full power, which can be confirmed by the slope of the curve in Figure 1. However, after the warm-up period, the controller outputs change frequently in the range $[-100,100]$ or $[0,100]$. It is highly possible that the $1 \mathrm{~Hz}$ sampling rate is not able to capture all the changes, and thus the resultant energy is lower than the calculated value.

By using the proposed method, it is also possible to monitor the energy consumption in different heating zones. According to the recorded data, Zone 1 consumes nearly half of the total thermal energy. This can be caused by the plastic granules absorbing heat energy when passing through Zone 1. However, due to the high heat conduction of metal, a significant amount of energy is wasted in cancelling the Zone 1 heating and feed area water cooling. The installation of a heat isolation plate between Zone 1 and the feed section should help to significantly reduce the overall thermal energy consumption.

\section{Monitoring of motor energy consumption}

The Killion KTS-100 extruder is fitted with Eurotherm 512C motor drive and a tacho meter. The motor controller also utilizes PID control algorithm implemented through PWM. For ease of configuration, the $512 \mathrm{C}$ controller also provides several terminals which can be used to read or write the motor status through analog or digital signals. These include the screw speed, setpoint ramp, tacho feedback, torque/current limit, current meter output, start/stop, and so on.

For a DC motor, the rotational speed is known to be proportional to the motor armature voltage while the screw torque is proportional to the motor armature current. This relationship can be summarized as

$$
\begin{gathered}
V_{\mathrm{a}}=R_{\mathrm{a}} I_{\mathrm{a}}+E_{\mathrm{b}} \\
E_{\mathrm{b}}=K_{v} \omega \\
T=K_{\mathrm{m}} I_{\mathrm{a}}
\end{gathered}
$$

where

- $\quad V_{\mathrm{a}}$ : Motor armature voltage or motor supply voltage.

- $I_{\mathrm{a}}$ : Motor armature current or motor supply current.

- $R_{\mathrm{a}}$ : Motor terminal resistance.

- $E_{\mathrm{b}}$ : Back electromotive force (EMF).

- $\omega$ : Motor rotational speed.

- $T$ : Motor torque.

- $K_{v}, K_{\mathrm{m}}$ : Motor-specific parameters which can be identified through the measurement of $V_{\mathrm{a}}, I_{\mathrm{a}}, T$, and $\omega$.

Conventionally, the power consumption can be simply obtained through the production of armature voltage and current. However, as PWM regulation is adopted, the voltage and current change frequently during each PWM cycle. This change causes a phase shift between the voltage and current, leading to a low power factor for this motor drive system. An additional power meter attached to the motor drive power supply can verify this effect. As a result, the DC motor consumes more energy than it actually requires to drive the screw. The recorded data also shows a clear relationship between the power factor and screw speed. Thus, a full representation of the motor apparent power consumption can be obtained.

\section{Melt quality monitoring}

Melt viscosity is regarded as one of the best quality indicators of the melt stream. It can be described as 'the resistance of material to flow', and is derived from the ratio of the shear stress and shear rate of the flow as

$$
\eta=\frac{\tau}{\dot{\gamma}}
$$

where $\eta$ represents the viscosity, $\tau$ is the shear stress, and $\dot{\gamma}$ denotes the shear rate. Under laboratory conditions, shear stress can be determined from the pressure drop along the channel of a slit die or capillary die (Liu et al., 2012), while shear rate can be calculated from the volumetric flow rate through the die. Another development in this area is known as a 'torsional viscometer' (http://www.manscoproducts.com/ index.htm, Venkatraman and Okano, 1990). Although this equipment can be used to continuously and accurately measure the melt viscosity, it is not applicable to industrial conditions due to its restriction of the melt stream, therefore a soft-sensor approach based on mathematical models becomes an affordable alternative. In the following, the fundamentals of slit die viscometry will be introduced. The recorded data from this viscometry will be used to train an RBF network model, providing indirect measurement of melt viscosity. 


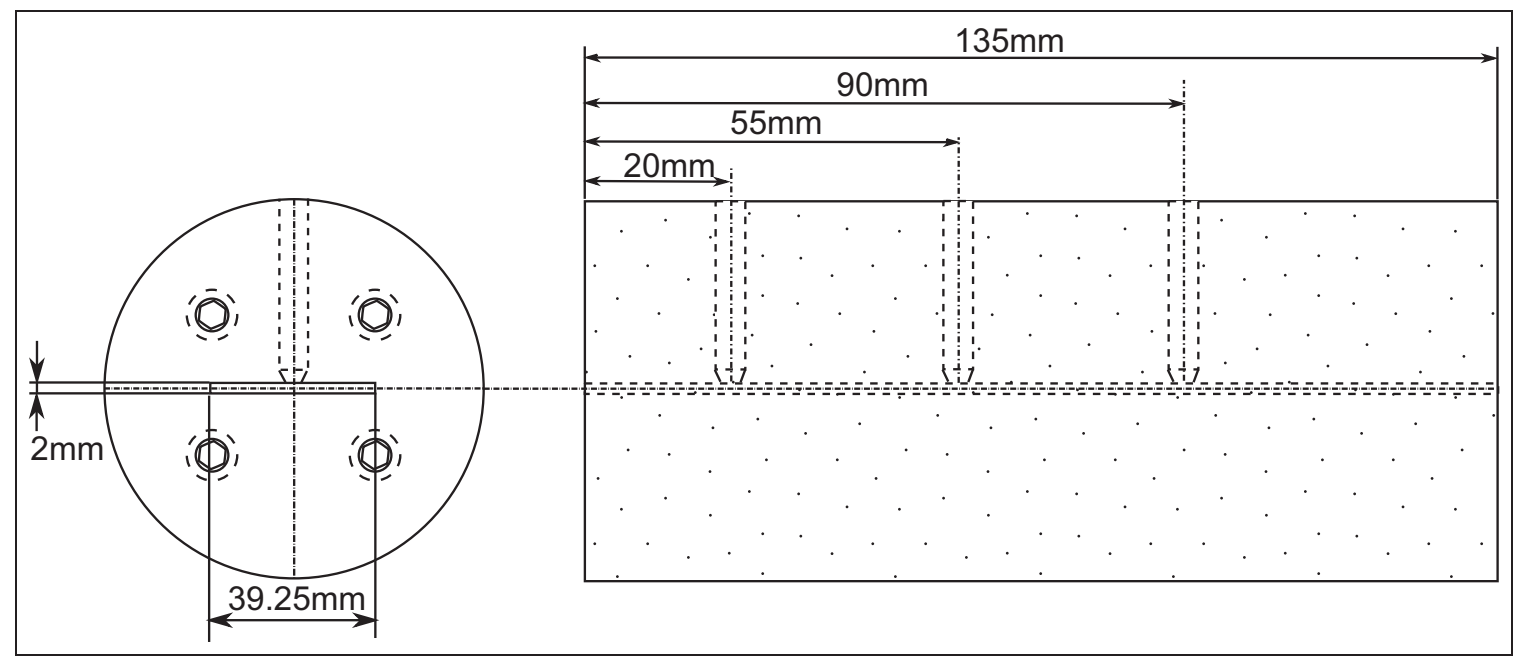

Figure 2. The structure of a slit die.

\section{Viscosity monitoring through a slit die}

In-line viscometer die. A slit-die rheometer is an extruder die which has a rectangular flow channel with a large width-to-height ratio (Figure 2). The apparent shear rate at the wall in a slit die can be determined by

$$
\dot{\gamma}_{\mathrm{aw}}=\frac{6 \dot{V}}{H^{2} W}
$$

where $\dot{V}$ denotes the flow rate through the flow channel, $H$ is the slit height and $W$ is the slit width $(W \gg H)$.

However, for a power law fluid with power law index $n$, the actual shear rate at the wall is (Rauwendaal, 2001)

$$
\dot{\gamma}_{\mathrm{w}}=\frac{2 n+1}{3 n} \dot{\gamma}_{\mathrm{aw}}
$$

The shear stress at the wall can be determined from the pressure drop along the channel

$$
\tau_{\mathrm{w}}=\frac{\Delta P}{L} \frac{H}{2}
$$

where $L$ is the distance between two pressure measurement points.

The actual viscosity of a power law fluid can then be obtained by

$$
\eta=\frac{n H^{3} W}{4(2 n+1) V} \frac{\Delta P}{L}
$$

Throughput measurement. The throughput is mainly determined by the melt pressure. Theoretically, a precision scale can be used below the slit die for continuous measurement of the mass throughput. However, large variations can be observed on the scale reading due to the elasticity of the polymer melt stream. As a result, the melt polymer was collected manually at 1 min intervals. The collected polymer was then weighed to obtain the mass throughput. The measurement error was minimized by taking the average value from multiple samples.

By plotting the mass throughput against screw speed, a strong linear relationship can be observed. As shown in Figure 3, the mass throughput is nearly equivalent to the screw speed minus one. The change of material from low density polyethylene (LDPE) $2102 \mathrm{TN} 32 \mathrm{~W}$ (melt flow rate (MFR) of $2.5 \mathrm{~g} / 10 \mathrm{~min}$ at $190^{\circ} \mathrm{C}$ and $2.16 \mathrm{~kg}$, density $921 \mathrm{~kg} / \mathrm{m}^{3}$ ) to LDPE 2102TN00W (MFR of $0.33 \mathrm{~g} / 10 \mathrm{~min}$ at $190^{\circ} \mathrm{C}$ and $2.16 \mathrm{~kg}$, density $921 \mathrm{~kg} / \mathrm{m}^{3}$ ) did not affect this linear relationship much. A similar relationship for mass throughput can also be found in McAfee (2005).

In order to get the volumetric flow rate, the melt density is also required. This was measured by an RH7 (Figure 4) viscometer under different temperature settings. During the test, a specific volume of melted polymer was pushed by a piston through a capillary die and collected for weighing. Variations as large as $50 \mathrm{~kg} / \mathrm{m}^{3}$ could be observed in the readings for both materials. This may be caused by the compression of the polymer during the test. A typical value of $762 \mathrm{~kg} / \mathrm{m}^{3}$ was chosen as the melt density for both materials. The error did not affect the viscosity stability analysis, only the actual viscosity reading.

Power law index. The power law index indicates the degree of shear thinning. Generally, the viscosity at either very low shear rates or very high shear rates is nearly constant. According to Rauwendaal (2001), the fluid behaves as a Newtonian fluid at either low or high shear rates. However, for most polymer processing applications, such as compression, molding, extrusion, and injection molding, the shear rate is in the range $\left[10^{0} 10^{4}\right]$. Within this range, the relationship between viscosity and shear rate can be reasonably approximated by a straight line in a log-log plot and given by 


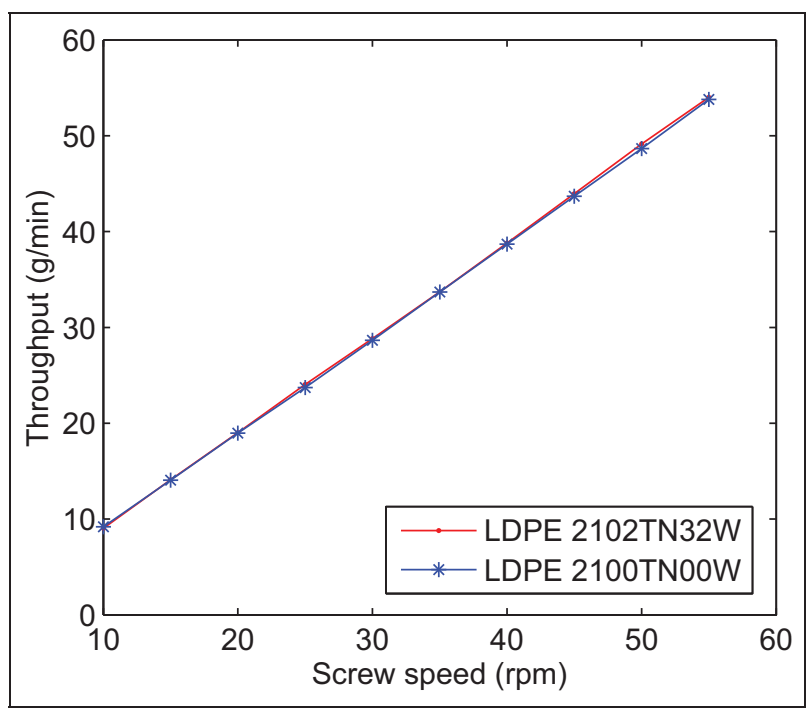

Figure 3. Throughput measurement with different LDPEs, linear approximation $Q=N-I(N \geq 5)$ with an $R$-squared value of 0.9999 .

$$
\eta=m \dot{\gamma}^{n-1}
$$

where $m$ is the consistency index and $n$ is the power law index.

For the viscosity calculation in equation (11), the power law index is an unknown parameter which must be determined before calculating the melt viscosity for each material. This is again achieved by using the RH7 viscometer, shown in Figure 4, where a long capillary die and a zero length orifice die are used together to determine the pressure drop along the capillary flow channel. In order to rectify the data for the non-Newtonian character of the melt, the Rabinowitsch correction or Bagley correction can be adopted. In this paper, Rabinowitsch correction is used to obtain the power law index for each material.

Two different materials were tested under different melting temperatures. The resultant viscosity against shear rate is shown in Figure 5. The power law index varies as the shear rate varies from low to high, thus the average value within the linear range was used. As shown in Table 1, higher temperatures lead to a somewhat higher power law index. The values obtained were used for the viscosity calculation.

\section{Viscosity monitoring through a soft-sensor approach}

While viscometers are widely used in laboratory conditions, they cannot be applied to industry manufacturing lines due to their restriction to the main melt stream. Alternatively, a model-based soft-sensor approach provides an achievable solution for industrial applications (Liu et al., 2012). The reliability of this method depends on the accuracy of the mathematical model. Therefore, a compact model with satisfactory generalization performance is desired. Although the models presented in Liu et al. (2012) show excellent approximation performance, the dynamics in some regions are missing, which may cause problems in future prediction. This paper fully excites the system inputs over a wider range for a longer period of time ( 2 hours instead of 20 minutes). It also adopts an RBF network because of its universal

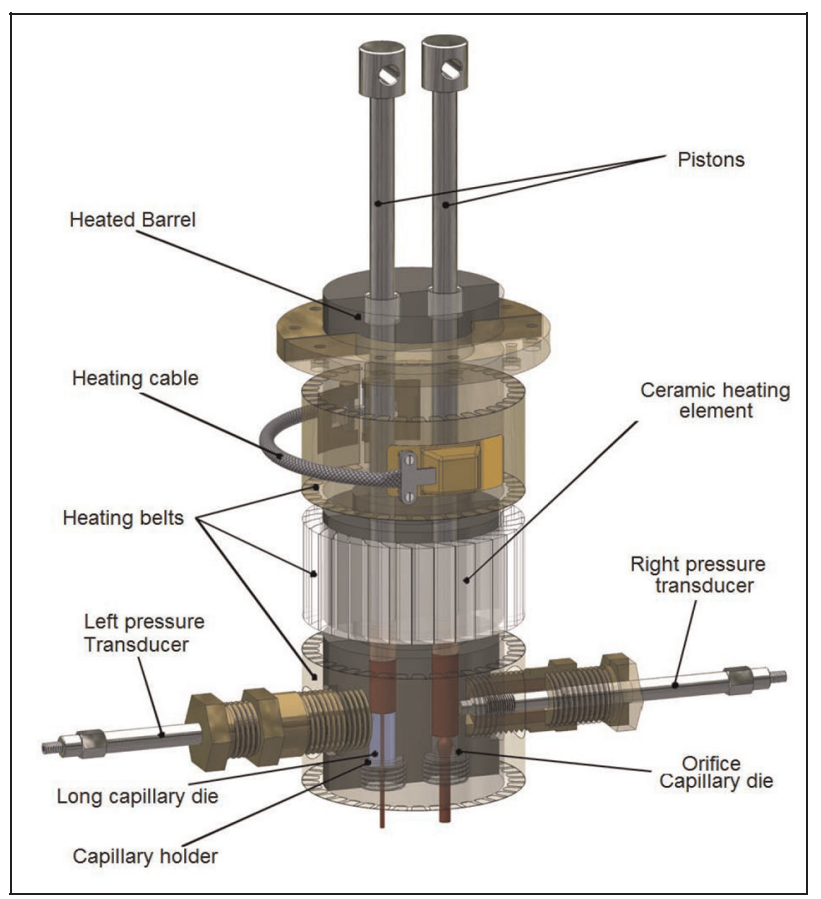

Figure 4. Structure of an $\mathrm{RH} 7$ rheology viscometer. Reproduced with kind permission from Elsevier (Zatloukal and Musil, 2009).

approximation capability. The non-linear parameters of the RBFs are optimized through differential evolution, while the number of hidden nodes and output weights are tackled by a two-stage selection algorithm (Li et al., 2006).

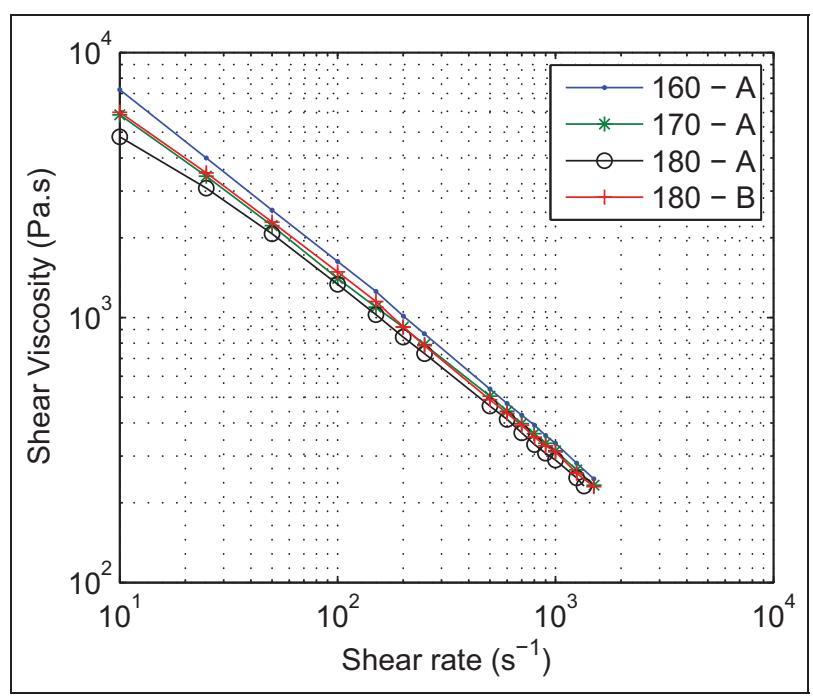

Figure 5. Shear viscosity function obtained using the RH7 rheology viscometer. (160, 170 and 180 are different barrel temperature settings in degree Celsius, and $A$ or $B$ means two different materials where $A$ : LDPE 2102TNOOW, MFR 0.33g/10min (at $190^{\circ} \mathrm{C}$ and $2.16 \mathrm{~kg}$ ), density $92 \mathrm{I} \mathrm{kg} / \mathrm{m}^{3}$; B: LDPE $2102 T N 32 \mathrm{~W}$, MFR $2.5 \mathrm{~g} / 10 \mathrm{~min}$ (at $190^{\circ} \mathrm{C}$ and 2.16 $\mathrm{kg})$, density $921 \mathrm{~kg} / \mathrm{m}^{3} \$$ ) 
Table I. Power law indices obtained using the RH7 rheology viscometer.

\begin{tabular}{lll}
\hline Material & Melting temperature & Power law index \\
\hline LDPE 2102TN00W & $160^{\circ} \mathrm{C}$ & 0.32 \\
LDPE 2102TN00W & $170^{\circ} \mathrm{C}$ & 0.36 \\
LDPE 2102TN00W & $180^{\circ} \mathrm{C}$ & 0.38 \\
LDPE 2102TN32W & $180^{\circ} \mathrm{C}$ & 0.46 \\
\hline
\end{tabular}

Radial basis function network model. A general RBF neural model can be expressed as

$$
y(t)=\sum_{k=1}^{n} \theta_{k} \varphi_{k}\left(\mathbf{x}(t) ; \mathbf{c}_{k} ; \mathbf{\Sigma}_{k}\right)+\varepsilon(t)
$$

where $y(t)$ is the actual output at sample time $t, \mathbf{x}(t) \in \Re^{p}$ is the input vector, $\varphi_{k}\left(\mathbf{x}(t) ; \mathbf{c}_{k} ; \mathbf{\Sigma}_{k}\right)$ denotes the non-linear activation function, $\mathbf{c}_{i}=\left[c_{i 1}, c_{i 2}, \cdots, c_{i p}\right]^{\mathrm{T}}$ is the centre vector, and $\boldsymbol{\Sigma}_{i}$ is the associated norm matrix. Finally, $\theta_{k}$ represents the output layer weight for each RBF node, and $\varepsilon(t)$ is the network error at sample time $t$. By using a set of $N$ data samples $\{\mathbf{x}(t), y(t)\}_{t=1}^{N}$ for model training, equation (13) can then be re-written in a matrix form as

$$
\mathbf{y}=\boldsymbol{\Phi}_{n} \boldsymbol{\theta}_{n}+\mathbf{e}
$$

If the regression matrix $\boldsymbol{\Phi}_{n}$ is of full column rank, the least-squares estimate of the regression coefficients in equation (14) is given by

$$
\hat{\boldsymbol{\theta}}=\left(\boldsymbol{\Phi}_{n}^{\mathrm{T}} \boldsymbol{\Phi}\right)_{n}^{-1} \boldsymbol{\Phi}_{n}^{\mathrm{T}} \mathbf{y}
$$

where $\boldsymbol{\Phi}_{n}^{\mathrm{T}} \boldsymbol{\Phi}_{n}$ is sometimes called the 'information matrix'. The associated minimal cost function is

$$
J_{n}\left(\hat{\boldsymbol{\theta}}_{n}\right)=\mathbf{y}^{\mathrm{T}}\left(\mathbf{I}-\boldsymbol{\Phi}_{n}\right)\left(\boldsymbol{\Phi}_{n}^{\mathrm{T}} \boldsymbol{\Phi}_{n}\right)^{-1} \boldsymbol{\Phi}_{n}^{\mathrm{T}} \mathbf{y}
$$

Differential evolution. In the RBF model construction process, the non-linear parameters, such as the centre c, and width matrix $\mathbf{\Sigma}$ are difficult to determine. Traditionally, the data samples are used as RBF centres while the width for each hidden node is obtained through exhaustive search or pre-determined by prior knowledge. Fortunately, metaheuristic optimization methods, such as simulated annealing (SA) (Kirkpatrick, 1984), evolutionary algorithm (EA) (Michalewicz, 1996), Tabu search (TS) (Glover and Marti, 2006), particle swarm optimization (PSO) (Kennedy and Eberhart, 1995), differential evolution (DE) (Storn and Price, 1997), and harmony search (HS) (Loganathan, 2001), can be adopted to find out the optimal non-linear parameters for RBF. In this application, both PSO and DE have been incorporated and the latter produced smaller training and testing errors, hence were introduced below.

As a population-based optimization technique, DE (Storn and Price, 1997) starts with some initial points which are randomly generated in the search space, and then pushes the population towards the global optimum point through repeated operations of mutation, crossover and selection. New populations are obtained by adding the weighted difference of two vectors to a third one, where the vectors are mutually different random points from the last generation.

Suppose $\mathbf{x}_{i}^{(l)}(i=1,2, \ldots, S)$ is a solution vector in generation $l$, and $\mathrm{S}$ denotes the population size. The operations in the classic DE method can be summarized as follows

- mutation: a mutant vector is generated by

$$
\mathbf{v}_{i}^{(l+1)}=\mathbf{x}_{r 1}^{(l)}+F\left(\mathbf{x}_{r 2}^{(l)}-\mathbf{x}_{r 3}^{(l)}\right)
$$

where $r 1, r 2, r 3$ are random indices from $[1,2, \ldots, S]$ and $F \in(0,2]$ is a real constant which controls the amplification of the added differential variation. Larger values of $F$ lead to higher diversity in new populations, while lower values cause faster convergence.

- crossover: this operation is implemented to increase the diversity of the population. A trial vector is defined as

$$
\mathbf{u}_{i}^{(l+1)}=\left[u_{i 1}^{(l+1)}, u_{i 2}^{(l+1)}, \cdots, u_{i p}^{(l+1)}\right] \mathrm{T}
$$

with elements given by

$$
u_{i j}^{(l+1)}= \begin{cases}v_{i j}^{(l+1)} & \operatorname{rand}_{i}(0,1) \leq C_{\mathrm{r}} \text { or } i=b_{\mathrm{r}} \\ x_{i j}^{(l)} & \text { otherwise }\end{cases}
$$

where $p$ is the dimension of a solution, $C_{\mathrm{r}} \in(0,1]$ is the pre-defined crossover constant, $\operatorname{rand}_{i}(0,1)$ uniformly generates a scaler from $(0,1]$ at the $i^{\text {th }}$ evaluation, and $b_{\mathrm{r}}$ is a random index chosen from $[1,2, \ldots, p]$ so that $\mathbf{u}_{i}^{(l+1)}$ contains at least one parameter from $\mathbf{v}_{i}^{(l+1)}$.

- selection: the last step is to compare all the trial vectors $\mathbf{u}_{i}^{(l+1)}$ with the target ones $\mathbf{x}_{i}^{(l)}$ according to a criterion, such as their contribution to a loss function, and then decide which one becomes a member of the next generation.

The above procedure continues until a pre-set number of iterations is reached or the desired accuracy is obtained.

Two-stage selection based on heuristic optimization. The twostage selection algorithm includes a forward model construction stage and a backward model refinement stage. During the first stage, one RBF centre is selected and added to the model at each step. The significance of each centre is measured by its contribution to the reduction of the cost function. This process continues until some pre-defined modelling criteria are met, the algorithm then moves to the second stage where the importance of previously selected centres is reviewed, and any insignificant ones are replaced. The computational efficiency involved in this process is achieved by defining a residual matrix $\mathbf{R}_{k}$ which can be updated recursively. Unlike conventional two-stage selection where the candidate terms are from the data samples, 
in this paper, DE is used to randomly generate a population and the global best solution is selected at each step.

With reference to equation (16), a recursive matrix $\mathbf{M}_{k}$ and a residual matrix $\mathbf{R}_{k}$ are defined to simplify the computation

$$
\begin{gathered}
\mathbf{M}_{k} \triangleq \mathbf{P}_{k}^{T} \mathbf{P}_{k} \quad k=1, \cdots, n \\
\mathbf{R}_{k} \triangleq I-\mathbf{P}_{k} \mathbf{M}_{k}^{-1} \mathbf{P}_{k}^{T} \quad \mathbf{R}_{0} \triangleq I
\end{gathered}
$$

where $\mathbf{P}_{k}^{T} \in \Re^{N \times k}$ contains the first $k$ columns of the regression matrix $\boldsymbol{\Phi}$ in equation (14).

By substituting equation (21) into equation (16), the cost function becomes

$$
J\left(\mathbf{P}_{k}\right)=\mathbf{y}^{T} \mathbf{R}_{k} \mathbf{y}
$$

At the first stage, the RBF centres are optimized one at a time, and given by the global best solution from the entire population of the heuristic methods after several iterations. Suppose at the $k^{\text {th }}$ step, one more centre $\mathbf{p}_{k+1}$ is to be added. The net contribution of $\mathbf{p}_{k+1}$ to the reduction of cost function can then be calculated as $\mathrm{Li}$ et al. (2005)

$$
\Delta J_{k+1}\left(\mathbf{P}_{k}, \mathbf{p}_{k+1}\right)=\frac{\left(\mathbf{y}^{\mathrm{T}} \mathbf{p}_{k+1}^{(k)}\right)^{2}}{\mathbf{p}_{k+1}^{\mathrm{T}} \mathbf{p}_{k+1}^{(k)}}
$$

where $\mathbf{p}_{k+1}^{(k)} \triangleq \mathbf{R}_{k} \mathbf{p}_{k+1}$. According to Li et al. (2005), an auxiliary matrix $\mathbf{A} \in \Re^{n \times n}$ and a vector $\mathbf{b} \in \Re^{n \times 1}$ need to be defined to reduce the computational complexity. Their elements are given by

$$
\begin{gathered}
a_{i, j} \triangleq\left(\mathbf{p}_{i}^{(i-1)}\right)^{\mathrm{T}} \mathbf{p}_{j}, \quad 1 \leq i \leq j \\
b_{j} \triangleq\left(\mathbf{p}_{j}^{(j-1)}\right)^{\mathrm{T}} \mathbf{y}, \quad 1 \leq j \leq n
\end{gathered}
$$

where $\left(\mathbf{p}_{j}^{(0)}=\mathbf{p}_{j}\right)$. The efficiency of the this forward stage then follows from updating these terms recursively as

$$
a_{i, j}=\mathbf{p}_{i}^{\mathrm{T}} \mathbf{p}_{j}-\sum_{l=1}^{i-1} a_{l, i} a_{l, j} / a_{l, l}
$$

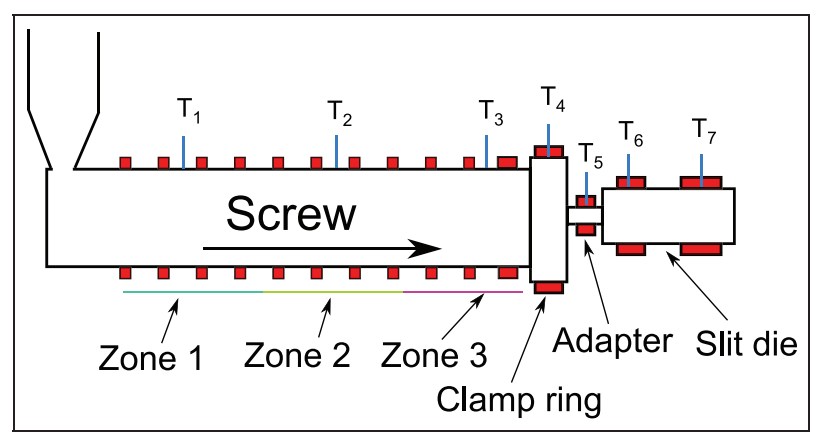

Figure 6. Seven heaters are installed on the KTS-I00 single-screw extruder.

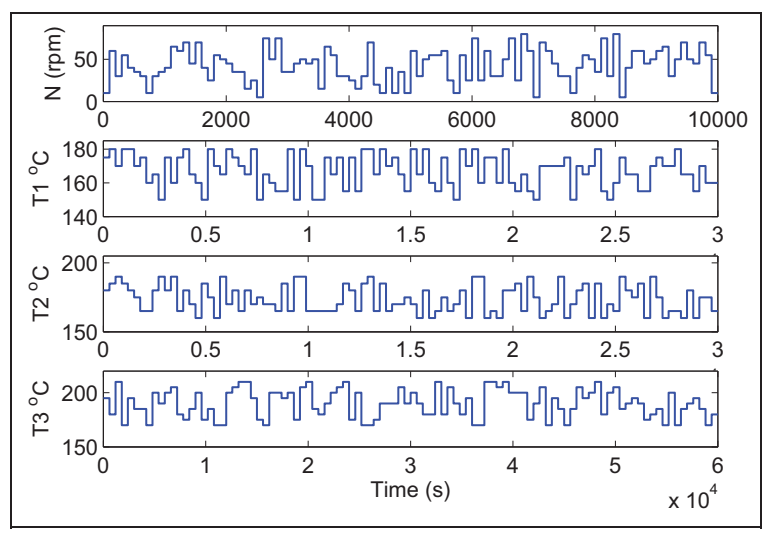

Figure 7. Excitation signal (PRS) for experiments and data collection.

$$
b_{j}=\mathbf{p}_{j}^{\mathrm{T}} \mathbf{y}-\sum_{l=1}^{j-1}\left(a_{l, j} b_{l}\right) / a_{l, l}
$$

By substituting equations (24) and (25) into equation (23), the net contribution of a new RBF centre $\mathbf{p}_{k+1}$ to the cost function can then be expressed as

$$
\Delta J_{k+1}\left(\mathbf{p}_{k+1}\right)=\frac{b_{k+1}^{2}}{a_{k+1, k+1}}
$$

In heuristic methods, equation (28) provides the formula to evaluate each solution in the population. For instance, the selection of local best and global best particles in PSO or the selection between trial vector and target vector in DE.

The model from the first stage was not optimal due to the correlations between selected terms. In other words, the prior selected centres introduced a constraint on the latter selections. A second stage was therefore adopted to eliminate this constraint and replace any insignificant centres by a new one generated from the population. As the last selected centre in the forward construction was always optimized for the whole network, the backward refinement can be divided into two main steps: firstly, a previously selected centre $\mathbf{p}_{k}, k=1, \ldots, n-1$ is shifted to the $n^{\text {th }}$ position as if it was the last selected one, then the DE is implemented to find an alternative centre at the $n^{\text {th }}$ position based on the re-ordered $n-1$ centres. If the shifted one is less significant than the new centre from the population, it will be replaced, leading to a reduced training error and potential improvement in the generalization capability. This review is repeated until a pre-defined number of check loops is reached. The detailed algorithm and its computational analysis can be found in Deng et al. (2011).

Modelling of melt viscosity. In order to properly stimulate the extrusion system to extract useful information for model training, a PRS signal was designed for each input, including screw speed and barrel heatings. In order to maintain a consistent viscosity from the adapter to the slit die, the temperature settings for heaters T3-T7 (see Figure 6) were kept the same. The PRS signal was generated in Matlab, and later imported to Labview. As shown in Figure 7, the screw speed was adjusted in a wide range from 5 to $75 \mathrm{rpm}$ while the temperature 
Table 2. Estimated parameters for the RBF network using two-stage selection and differential evolution.

\begin{tabular}{|c|c|c|c|}
\hline Hidden node & Centre vector & Width vector & Output weights \\
\hline I & {$[0.435,0.014,0.239,0.542,0.248,0.110]$} & {$[0.897,0.380,0.44 \mathrm{I}, 0.244,0.766,0.6 \mathrm{II}]$} & -0.771 \\
\hline 2 & {$[0.452,1.000,0.683,0.573,1.000,0.582]$} & {$[0.148,0.914,1.000,0.025,0.427,0.422]$} & 0.217 \\
\hline 3 & {$[0.726,0.171,0.024,1.000,0.196,0.372]$} & {$[0.148,0.194,0.152,0.777,0.630,0.328]$} & -4.255 \\
\hline 4 & {$[0.714,0.412,0.000,0.434,0.127,0.000]$} & {$[0.379,0.548,0.664,0.020,0.093,0.506]$} & $1.46 \mathrm{I}$ \\
\hline 5 & {$[0.014,0.574,0.556,0.247,0.938,0.715]$} & {$[0.166,0.565,1.000,0.452,1.000,0.185]$} & 4.622 \\
\hline 6 & {$[0.152,0.014,0.787,0.504,0.000,0.000]$} & {$[0.242,0.701,0.715,0.149,0.367,1.000]$} & 0.474 \\
\hline 7 & {$[0.014,0.547,0.608,0.234,0.223,0.112]$} & {$[0.162,0.710,0.550,0.872,0.961,0.045]$} & 0.436 \\
\hline 8 & {$[0.014,0.349,0.000,0.000,0.000,0.000]$} & {$[1.000,0.93 \mathrm{I}, 0.880,0.993,0.622,0.83 \mathrm{I}]$} & 0.876 \\
\hline
\end{tabular}

settings for the barrel heating were changed around the equilibrium points. The viscosity data was obtained through the slit die described above. By substituting the geometry parameters into equation (11), the viscosity can be calculated as

$$
\begin{aligned}
\eta & =\frac{0.03925 \times 0.002^{3} \times 762 \times 60 \Delta P n}{4 \times 0.35(1.01 N-1.416)(2 n+1)} \\
& =10254 \times 10^{-5} \times \frac{n}{2 n+1} \frac{\Delta P}{(1.01 N-1.416)}
\end{aligned}
$$

where a typical melt density of $762 \mathrm{~kg} / \mathrm{m}^{3}$ is adopted for LDPE. According to equation (29), the viscosity is very sensitive to melt pressure. A variation of $0.1 \mathrm{MPa}$ in pressure may lead to a 16 Pas change in viscosity. Thus, it is necessary to filter out noise in the pressure. There is also a signal delay between two measuring points along the slit die, which can be compensated with a low-pass Butterworth filter.

The sampling rate was set at $10 \mathrm{~Hz}$, and the extruder ran continuously for about $2 \mathrm{~h}$ after a start-up period of $30 \mathrm{~min}$. For model training and validation, the collected data was down sampled to $1 \mathrm{~Hz}$. Therefore, a total of 9931 data points was used, with the first two thirds used for model training and the rest reserved for model validation.

The melt pressure $P_{\text {melt }}$, melt temperature $T_{\text {melt }}$, and armature current $I_{\text {motor }}$ of the screw driving motor were selected as model inputs. The dynamic orders were pre-set at 2 for each input, and their corresponding delays were pre-determined at $[1,20,1]$ based on prior knowledge. Without losing any nonlinear dynamics, the collected data were re-scaled to the range of $[0,1]$ for each input and output.

The amplification coefficient $F$ in equation (17) was set at 0.8 , while 0.6 was chosen as the crossover constant $C_{\mathrm{r}}$ in equation (19). The population size of DE for this application was pre-set at 30 , and 30 updating cycles were implemented to find an optimal solution. As a stochastic optimization method, this process cannot produce the same result for each run, thus the best model from 10 runs was selected for viscosity modelling. The model parameters obtained are given in Table 2.

The model approximation performance is illustrated in Figure 8. Most of the errors are within the range of $[0,200]$.

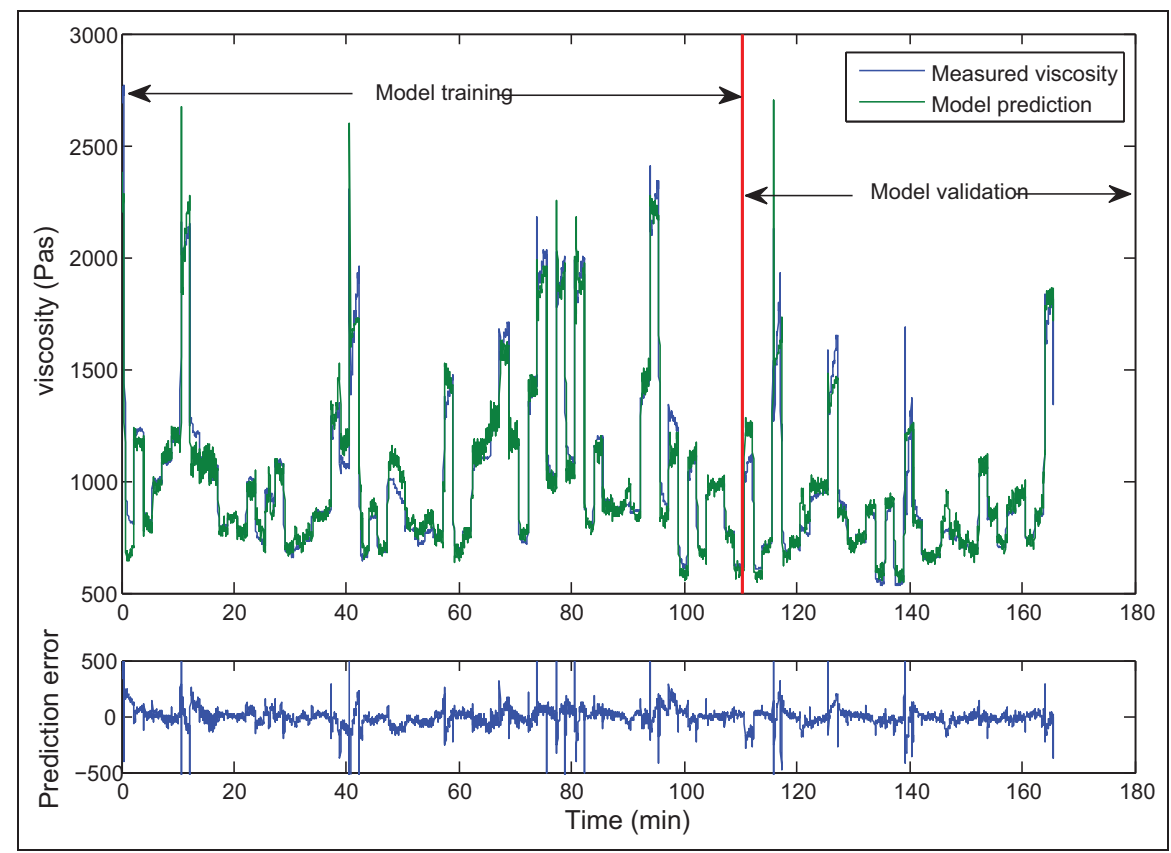

Figure 8. Approximation performance of developed RBF model for melt viscosity. 
The root mean square percentage error (RMSPE) on the test data was $9.35 \%$. Because the training data covers a wide operating range, the resultant model shows satisfactory generalization performance on new data.

\section{Conclusions and future work}

Simple and efficient process monitoring methods for polymer extrusion have been developed in this paper. For energy consumption, the new methods not only monitor the thermal energy usage profile along the extruder, but also provide accurate and reliable monitoring of both thermal energy and motor drive energy consumption, independent of extruder geometry and material. For melt quality, the model-based soft-sensor approach provides an satisfactory alternative to the slit-die rheometer for measuring viscosity. The integration of differential evolution into a two-stage model selection process enabled a compact and accurate model to be generated.

Future work will involve validation of the proposed monitoring methods on a variety of extruders and other polymer processing units. The RBF model for viscosity monitoring could also be improved by incorporating optimal experimental design criteria and other optimization approaches, such as harmony search. Instead of using a complex non-linear model to approximate melt viscosity, local linear models will also be investigated to model the extrusion process.

\section{Funding}

This work was supported by the Engineering and Physical Sciences Research Council (grant number EP/G059489/1), an Invest NI proof-of-concept grant (number POC333), and the Science and Technology Commission of Shanghai Municipality (grant number 11ZR1413100).

\section{References}

Abeykoon C, McAfee M, Li K, Martin P, Deng J and Kelly A (2010) Modelling the effects of operating conditions on motor power consumption in single screw extrusion. In: Li K, Fei M, Jia L and Irwin GW (eds) Life System Modeling and Intelligent Computing. New York: Springer, vol. 6329, pp. 9-20.

Cogswell F (1981) Polymer Melt Rheology: A Guide for Industrial Practice. London, UK: George Godwin Ltd, p. 178.
Davis P (2011) Challenges for the British plastics industry. Technical report. Cambridge, UK: British Plastics Federation.

Deng J, Li K, Irwin G and Fei M (2011) Two-stage RBF network construction based on PSO. Transactions of the Institute of Measurement and Control 35(1): 25-33.

Glover F and Marti R (2006) Tabu search. Metaheuristic Procedures for Training Neutral Networks 36: 53-69.

Kelly A, Brown E and Coates P (2006) The effect of screw geometry on melt temperature profile in single screw extrusion. Polymer Engineering \& Science 46: 1706-1714.

Kennedy J and Eberhart R (1995) Particle swarm optimization. In: IEEE international conference on neural networks, Perth, Australia, 27 November-1 December 1995, vol. 4, pp. 1942-1948. Piscataway: IEEE Press.

Kent R (2008) Energy management in plastics processing. Plastics, Rubber and Composites 2: 96-104.

Kirkpatrick S (1984) Optimization by simulated annealing: Quantitative studies. Journal of Statistical Physics 34: 975-986.

Lai E and Yu D (2000) Modeling of the plasticating process in a single-screw extruder: A fast-track approach. Polymer Engineering \& Science 40: 1074-1084.

Li K, Peng J and Irwin G (2005) A fast nonlinear model identification method. IEEE Transactions on Automatic Control 50: 1211-1216.

Li K, Peng JX and Bai EW (2006) A two-stage algorithm for identification of nonlinear dynamic systems. Automatica 42: 1189-1197.

Liu X, Li K and McAfee M (2012) Dynamic grey-box modelling for online monitoring of extrusion viscosity. Polymer Engineering \& Science 52: 1332-1341.

Liu X, Li K, McAfee M and Deng J (2010) 'Soft-sensor' for real-time monitoring of melt viscosity in polymer extrusion process. In: 49 th IEEE conference on decision and control, Georgia, USA, 15-17 December 2010, pp. 3469-3474. Piscataway: IEEE Press.

Loganathan G (2001) A new heuristic optimization algorithm: Harmony search. Simulation 76: 60-68.

McAfee M (2005) Soft sensor for viscosity control of polymer extrusion. PhD Thesis, Queen's University Belfast, UK.

Michalewicz Z (1996) Genetic Algorithms + Data Structures $=$ Evolution Programs. New York: Springer.

Rauwendaal C (2001) Polymer Extrusion. Ohio, USA: Hanser Gardner.

Storn R and Price K (1997) Differential evolution-a simple and efficient heuristic for global optimization over continuous spaces. Journal of Global Optimization 11: 341-359.

Venkatraman S and Okano M (1990) A comparison of torsional and capillary rheometry for polymer melts: The Cox-Merz rule revisited. Polymer Engineering \& Science 30: 308-313.

Zatloukal M and Musil J (2009) Analysis of entrance pressure drop techniques for extensional viscosity determination. Polymer Testing 28: 843-853. 